\title{
Inverse Perturbation Method for Structural Redesign with Frequency and Mode Shape Constraints
}

\author{
Curtis J. Hoff, ${ }^{*}$ Michael M. Bernitsas, $†$ Robert E. Sandström, $\ddagger$ and William J. Anderson§ \\ The University of Michigan, Ann Arbor, Michigan
}

\begin{abstract}
A procedure is described for the redesign of undamped structural systems to meet natural frequency and/or mode shape objectives. The procedure can be applied to large or small modal changes and is based on a single finite element analysis of the baseline system. Perturbation of the baseline system is used to develop a set of equations that characterize the redesign process. Depending on the number of modal objectives and design variables, the problem is formulated as underconstrained, properly constrained, or overconstrained. All three problems are solved using an incremental predictor-corrector technique within the feasibility domain defined by the practical constraints imposed on the design variables. The procedure is illustrated by the redesign of a 1254 degree-of-freedom casting for a frequency objective.
\end{abstract}

\begin{tabular}{|c|c|}
\hline \multicolumn{2}{|r|}{ Nomenclature } \\
\hline$c_{i j}$ & $\begin{array}{l}=\text { participation of the } j \text { th mode to changes in } \\
\text { the } i \text { th mode }\end{array}$ \\
\hline$i$ & $=$ index associated with the $i$ th mode \\
\hline$j$ & $=$ index associated with the $j$ th mode \\
\hline$[K],\left[K^{\prime}\right]$ & $\begin{aligned}= & \text { generalized stiffness matrix of the baseline } \\
& \text { and objective structures, respectively }\end{aligned}$ \\
\hline$[k],\left[k^{\prime}\right]$ & $\begin{aligned}= & \text { stiffness matrix of the baseline and ob- } \\
& \text { jective structures, respectively }\end{aligned}$ \\
\hline$\left[k_{e}\right]$ & $=$ stiffness matrix of element $e$ \\
\hline$[M],\left[M^{\prime} \backslash\right]$ & $\begin{aligned}= & \text { generalized mass matrix of the baseline and } \\
& \text { objective structures, respectively }\end{aligned}$ \\
\hline$M_{i}$ & $\begin{array}{l}=\text { generalized mass of the } i \text { th mode of the } \\
\text { baseline structure }\end{array}$ \\
\hline$[m],\left[m^{\prime}\right]$ & $\begin{array}{l}=\text { mass matrix of the baseline and objective } \\
\text { structures, respectively }\end{array}$ \\
\hline$\left[m_{e}\right]$ & $=$ mass matrix of element $e$ \\
\hline$N$ & $\begin{aligned}= & \text { total number of increments in predictor- } \\
& \text { corrector solution }\end{aligned}$ \\
\hline$n$ & $\begin{aligned}= & \text { number of degrees of freedom in the } \\
& \text { structural model }\end{aligned}$ \\
\hline$S$ & $=$ number of modal objectives \\
\hline$s$ & $\begin{aligned} &= \text { number of modes involved in the redesign } \\
& \text { process }\end{aligned}$ \\
\hline[]$^{T},(1)^{T}$ & $=\begin{array}{l}\text { transpose of a matrix and vector, } \\
\text { respectively }\end{array}$ \\
\hline$\alpha_{e}$ & $=$ fractional change to element $e$ \\
\hline$\Delta$ & $=$ prefix denoting change \\
\hline$[\Delta k]$ & $=$ change to stiffness matrix \\
\hline$[\Delta m]$ & $=$ change to mass matrix \\
\hline$[\Delta \phi]$ & $=$ matrix of mode shape vector changes \\
\hline$\Delta\left(\omega_{i}^{2}\right)$ & $\begin{aligned}= & \text { change to the } i \text { th baseline structure } \\
& \text { eigenvalue } \omega_{i}^{2}\end{aligned}$ \\
\hline$\sigma$ & $=$ number of structural changes \\
\hline
\end{tabular}

Received May 15, 1983; revision received Dec. 20, 1983. Copyright (C) 1984 by Curtis J. Hoff. Published by the American Institute of Aeronautics and Astronautics with permission.

${ }^{*}$ Graduate Student Research Assistant, Department of Naval Architecture and Marine Engineering. Student Member AIAA.

†Assistant Professor, Department of Naval Architecture and Marine Engineering.

¥Adjunct Assistant Professor, Department of Naval Architecture and Marine Engineering (presently Research Engineer, Exxon Production Research Company). Member AIAA.

§Professor, Department of Aerospace Engineering. Member AIAA.

$$
\begin{aligned}
{[\phi],\left[\phi^{\prime}\right]=} & \text { matrix of mode shape vectors of the } \\
& \text { baseline and objective systems, respectively } \\
\{\psi\}_{i},\left\{\psi^{\prime}\right\}_{i}= & \text { th mode shape vector of }[\phi] \text { and }\left[\phi^{\prime}\right], \\
& \text { respectively } \\
= & k \text { th degree of freedom of the ith baseline } \\
& \text { mode shape } \\
= & i \text { th baseline and objective structure natural } \\
& \text { frequencies }
\end{aligned}
$$

\section{Introduction}

$\mathrm{N}$ many structural problems, the criteria for an acceptable design involve constraints on free vibration characteristics. The constraints may be on one or more of the natural frequencies, mode shapes, stress levels, or other structural characteristics. Several degrees of freedom may be constrained on any one mode. In most cases the first design does not satisfy all the free vibration objectives and/or practical constraints. Therefore, reanalysis of the structural system, which requires expensive finite element formulation and analysis, is necessary. Alternatively, the redesign procedure of the baseline system (first design), based on the perturbation technique proposed in this paper, can be used. This procedure does not require additional finite element analyses and can be applied to large or small modal changes. It is hereafter called the inverse perturbation redesign (IPR) procedure. The method can handle problems with both frequency and mode shape constraints. Direct limits on the design variables may also be specified.

Let $\sigma$ be the number of design variables in the IPR procedure, that is, the number of structural system particulars allowed to change during the redesign, and $S$ the number of modal objectives. If $s$ is the number of modes involved in the redesign procedure and the practical constraints, the problem can be reduced to one of the following types:

1) Underconstrained problem if $S<\sigma$. In this case the design is not unique and the problem can be formulated as an optimization problem. For this purpose an optimality criterion is needed, such as minimum structural weight (linear programming problem) or minimum change (quadratic programming problem) from the baseline system. The optimization variables in this problem are the $\sigma$ design variables in the IPR, plus $s \cdot n$ where $n$ is the number of structural degrees of freedom. The $S$ redesign objectives and the $s \cdot n$ free vibration equations become equality constraints in the optimization problem. Further, practical constraints in the form of inequalities may be imposed. 
2) Properly constrained problem if $S=\sigma$. In this case there are $S+s \cdot n$ equations that can be simultaneously solved for the $\sigma+s \cdot n$ unknowns, yielding either a finite number of structural designs or no solution. From the point of view of optimization, this is a constraint-bound problem that can produce a finite number of feasible designs or prove the problem to be infeasible. In the latter case the problem should be treated as in case 3 below.

3) Overconstrained problem if $S>\sigma$. In this case the $S+s \cdot n$ equations cannot be satisfied by the $\sigma+s \cdot n$ unknowns. Consequently, a minimum error criterion is needed in order to get a finite number of acceptable designs that will satisfy the equations and practical constraints within a minimum error.

\section{Background}

Historically, procedures involving natural frequency objectives were developed first. Since the first procedure developed by Rayleigh in 1873, many methods have been proposed. ${ }^{1}$ Only recently have methods aimed at solving the combined frequency and mode shape objective problems, such as the one presented in this paper, been developed.

Frequently, objective procedures usually are of the type of problem 1 described in the introduction, that is, their goal is to minimize the mass of a structure with specified frequency values or to maximize the frequency for a given total mass. Practical constraints are sometimes placed on design variables such as thickness of plates, cross-sectional area of axial bars, or moment of inerita of beams. Turner proposed one of the first methods to solve this problem. ${ }^{2}$ The free vibration equations were considered as equality constraints and handled using the Lagrange multiplier method. Taylor solved the problem for an axially vibrating bar by minimizing the total energy of the system using Hamilton's principle. ${ }^{3}$ Sheu extended the work of Turner and Taylor to situations where the number of constant stiffness segments are specified, but the boundaries and specific stiffness values of the segments were design variables in the minimum bar weight problem. ${ }^{4}$ For a more detailed review of many of these earlier methods, the reader is referred to the survey by Pierson. ${ }^{5}$

In more recent work, Taylor investigated the frequencyonly constrained problem in terms of model correlation. ${ }^{6} \mathrm{~A}$ procedure was developed to scale an existing structural model to meet experimentally measured natural frequencies. The modification scheme is based on the first-order terms of a Taylor series expansion about the baseline model. Bellagamba employed an exterior penalty function technique based on the first derivatives of the violated constraints. ${ }^{7}$ Additional constraints were imposed on static displacements and element stresses.

The problem of combined natural frequency and mode shape constraints has lately received considerable attention in terms of perturbation-based solution techniques. Stetson proposed a first-order perturbation method based on the assumption that the new mode shapes could be expressed as admixtures of the baseline mode shapes. ${ }^{8}$ In subsequent work, the technique was cast in terms of finite elements and was applied to several problems. ${ }^{9-11}$ Stetson's procedure, however, used a method of specifying mode shape constraints based on admixture coefficients that had no obvious physical interpretation. Sandström developed first-order equations similar to Stetson's, but provided a method for specifying mode shape constraints based on physical quantities. ${ }^{12,13} \mathrm{Kim}$ formulated the problem using the complete nonlinear perturbation equations. ${ }^{14} \mathrm{He}$ employed a penalty function method, where the objective function was a minimum weight condition and the penalty term was a set of residual force errors.

\section{Mathematical Formulation}

The undamped free vibration of a baseline discrete structural system is governed by the matrix equation

$$
[m]_{n \times n}\{\ddot{\psi}\}_{n \times l}+[k]_{n \times n}\{\psi\}_{n \times l}=\{0\}_{n \times l}
$$

An eigenvalue analysis produces the eigenvectors

$$
[\phi]=\left[\{\psi\}_{1},\{\psi\}_{2}, \ldots,\{\psi\}_{n}\right]
$$

and the natural frequencies

$$
\Gamma \omega^{2} \checkmark=\left[\begin{array}{cccc}
\omega_{I}^{2} & & & 0 \\
& \omega_{2}^{2} & & \\
& \bullet & \\
& & \bullet & \\
0 & & & \omega_{n}^{2}
\end{array}\right]
$$

Using the calculated eigenvectors the governing equations can be uncoupled and written as

$$
\left\lceil K \backslash=\left\lceil M \backslash\left\lceil\omega^{2}\right\rfloor\right.\right.
$$

where $[K]$ is the generalized stiffness matrix

$$
\left\lceil K \bigvee=[\phi]^{T}[k][\phi]\right.
$$

and $[M]$ is the generalized mass matrix

$$
\Gamma M J=[\phi]^{T}[m][\phi]
$$

Similarly, the uncoupled equations of an objective system are

$$
\left\ulcorner K^{\prime}\right\rfloor=\left[M ^ { \prime } \vee \left\lceil\omega^{\prime 2} \downarrow\right.\right.
$$

where $\left\lfloor K^{\prime}\right\rfloor$ is the objective system generalized stiffness matrix

$$
\left[K^{\prime} \checkmark=\left[\phi^{\prime}\right]^{T}\left[k^{\prime}\right]\left[\phi^{\prime}\right]\right.
$$

and $\left[M^{\prime} \checkmark\right.$ is the corresponding generalized mass matrix

$$
\left[M^{\prime} \vee=\left[\phi^{\prime}\right]^{T}\left[m^{\prime}\right]\left[\phi^{\prime}\right]\right.
$$

Relationships between the two systems can be defined in terms of perturbations of the baseline system, that is,

$$
\begin{gathered}
{\left[m^{\prime}\right]=[m]+[\Delta m]} \\
{\left[k^{\prime}\right]=[k]+[\Delta k]} \\
{\left[\omega^{\prime 2}\right]=\left[\omega^{2} \downarrow+\left[\Delta\left(\omega^{2}\right)\right]\right.} \\
{\left[\phi^{\prime}\right]=[\phi]+[\Delta \phi]}
\end{gathered}
$$

Through these definitions, the uncoupled equations (7) of the objective system can be rewritten in terms of the baseline system as

$$
\begin{gathered}
{\left[\phi^{\prime}\right]^{T}[\Delta k]\left[\phi^{\prime}\right]-\left[\phi^{\prime}\right]^{T}[\Delta m]\left[\phi^{\prime}\right]\left[\omega^{\prime 2}\right]} \\
=\left[\phi^{\prime}\right]^{T}[m]\left[\phi^{\prime}\right]\left[\omega^{\prime 2} \checkmark-\left[\dot{\phi}^{\prime}\right]^{T}[k]\left[\phi^{\prime}\right]\right.
\end{gathered}
$$

Equation (14) is called the general perturbation equation. It is nonlinear in terms of the modal quantities $\left[\phi^{\prime}\right]$ and $\left[\omega^{\prime 2}\right]$, but is linear with respect to the desired structural changes $[\Delta k]$ and $[\Delta m]$. To facilitate the solution of this equation, note that it is composed of $n^{2}$ scalar equations of the form

$$
\begin{aligned}
& \left\{\psi^{\prime}\right\}_{j}^{T}[\Delta k]\left\{\psi^{\prime}\right\}_{i}-\left\{\psi^{\prime}\right\}_{j}^{T}[\Delta m]\left\{\psi^{\prime}\right\}_{i} \omega_{i}{ }^{2} \\
& =\left\{\psi^{\prime}\right\}_{j}^{T}[m]\left\{\psi^{\prime}\right\}_{i} \omega_{i}{ }^{2}-\left\{\psi^{\prime}\right\}_{j}^{T}[k]\left\{\psi^{\prime}\right\}_{i}
\end{aligned}
$$

for $i, j=1,2, \ldots, n$. Equation (14) or (15) must be satisfied by the objective system and it should satisfy the $S$ modal objectives.

The structural change can be decomposed into $\sigma$ element change properties $\alpha_{e}$. In sheet metal or die cast systems, many 
elements must change together for manufacturability. In this case, $\sigma$ is the number of groups of perturbed elements. Then $[\Delta k]$ and $[\Delta m]$ become

$$
\begin{aligned}
& {[\Delta k]_{\text {system }}=\sum_{e=1}^{\sigma}\left[\Delta k_{e}\right]} \\
& {[\Delta m]_{\text {system }}=\sum_{e=1}^{\sigma}\left[\Delta m_{e}\right]}
\end{aligned}
$$

Elements not included in the $\sigma$ groups are not allowed to change. Furthermore, each element change can be expressed as a fractional change from the baseline system (or a sum of terms as needed to separate bending, stretching, and torsion) as

$$
\begin{aligned}
& {\left[\Delta k_{e}\right]=\left[k_{e}\right] \alpha_{e}} \\
& {\left[\Delta m_{e}\right]=\left[m_{e}\right] \alpha_{e}}
\end{aligned}
$$

These linear equations of $\alpha_{e}$ are used in the IPR procedure developed in this paper. Nonlinear relations can readily be implemented in the algorithm for other applications. For example, the effect of plate thickness on stretching stiffness is linear, while its effect on bending is of third order. The $\sigma$ change properties $\alpha_{e}$ are the design variables in the IPR. Finally, several of the design variables may be subject to practical constraints. These may be maximum or minimum size constraints or relative size constraints between the elements.

In problem 1, defined in the introduction, Eq. (14) or (15) and the $S$ modal objectives constitute equality constraints and the practical constraints constitute the inequality constraints. In problem 2, Eq. (14) or (15) and the $S$ modal objectives can be simultaneously solved for the $\sigma, \alpha_{e}$ that must fall in the feasibility domain defined by the practical constraints. In problem 3, Eq. (14) or (15) and the $S$ modal objectives can be satisfied along with the practical constraints within a specified error to yield the $\sigma, \alpha_{e}$.

\section{Solution Technique}

Solution of Eq. (14) or (15) will provide the required structural changes to meet the modal objectives. However, direct solution is usually not possible because of the implicit nature and complexity of these equations. The solution of the general perturbation equations (15) developed in this work is based on a predictor-corrector technique. The predictor phase uses the solution procedure for the first-order equation to provide a first approximation to the elemental changes $[\Delta k]$ and $[\Delta m]$. Using these approximate elemental changes, approximate objective eigenvectors are calculated. In the corrector phase, these eigenvectors are used in the general perturbation equations (15) to correct the elemental changes. The solution algorithm is diagrammatically shown in Fig. 1. In the case of large modal characteristic changes, the algorithm is applied in increments. In all of the applications we have tried, a single analysis of the baseline system has proved adequate. For very large systems, the result of the IPR method may not be as close as desired to the objective system. The algorithm may be improved by increasing the number of increments and/or by performing reanalysis at one or more intermediate steps. Either remedy will make the algorithm more expensive.

\section{Predictor Phase}

In the predictor phase of the solution algorithm, a firstorder solution to Eqs. (15) is required. The first-order equations developed here were originally proposed by Stetson and extended by Sandström. ${ }^{8-13}$ In the first-order perturbation development of Stetson, the mode shape changes

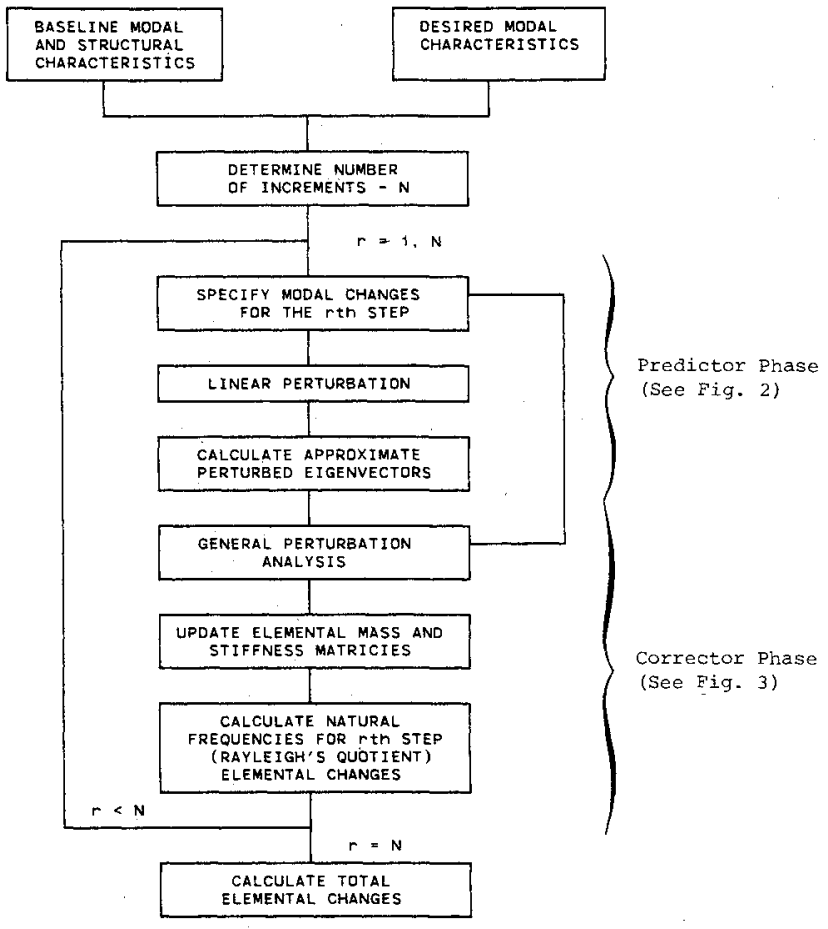

Fig. 1 Predictor-corrector solution technique.

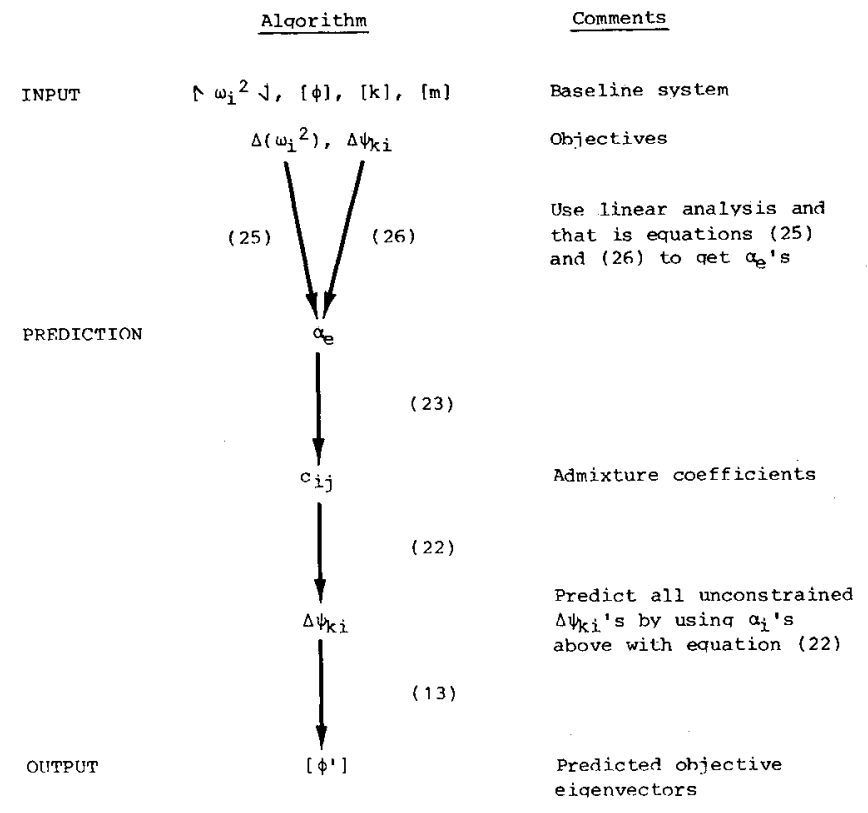

Fig. 2 Predictor phase algorithm.

were represented as linear combinations of the mode shapes obtained in the analysis of the baseline system

$$
[\Delta \phi]=[\phi][c]^{T}
$$

where the admixture coefficients $c_{i j}, i, j=1,2, \ldots, n$ are small and $c_{i i}=0$. This representation transforms the space spanned by the eigenvectors $\{\psi\}_{i}, i=1,2, \ldots, n$ into one that is numerically more convenient. Any method using such a transformation is an indirect search method since it searches for the admixture coefficient values instead of the eigenvector changes.

Applying this relationship for the eigenvector changes and neglecting terms of order $\Delta^{2}, \Delta^{3}$, and $\Delta^{4}$ we get the first-order 
perturbation equations in terms of $\Delta$

$$
\begin{aligned}
\{\psi\}_{j}^{T}[\Delta k]\{\psi\}_{i}-\{\psi\}_{j}^{T}[\Delta m]\{\psi\}_{i} \omega_{i}^{2} & =M_{i} \Delta\left(\omega_{i}^{2}\right) \quad \text { for } i=j \\
& =M_{j} c_{i j}\left(\omega_{i}^{2}-\omega_{j}^{2}\right) \text { for } i \neq j
\end{aligned}
$$

where $M_{i}$ and $M_{j}$ are the $i$ th and $j$ th generalized mass terms given by Eq. (6).

Solution of the first-order equations require the specification of the frequency changes $\Delta\left(\omega_{i}^{2}\right)$ and the mode shape changes $\Delta \psi_{k i}$ in terms of the admixture coefficients $c_{i j}$, where $\Delta \psi_{k i}$ is the change in the $k$ th degree of freedom of the $i$ th mode.

In order to eliminate the admixture coefficients, whose physical interpretation is difficult, the following algebraic manipulations are performed. Note that the change to the $k$ th degree of freedom of the $i$ th mode in terms of the admixture coefficients is

$$
\begin{gathered}
\Delta \psi_{k i}=c_{i 1} \psi_{k 1}+c_{i 2} \psi_{k 2}+\ldots+c_{i, i-1} \psi_{k, i-1}+c_{i, i+l} \psi_{k, i+l} \\
+\ldots+c_{i n} \psi_{k n}=\sum_{\substack{j=1 \\
j \neq i}}^{n} c_{i j} \psi_{k j}
\end{gathered}
$$

Also note that by rearranging Eq. (21) when $i \neq j$, the admixture coefficient $c_{i j}$ can be expressed as

$c_{i j}=\frac{1}{M_{j}\left(\omega_{i}^{2}-\omega_{j}^{2}\right)}\left(\{\psi\}_{j}^{T}[\Delta k]\{\psi\}_{i}-\omega_{i}^{2}\{\psi\}_{j}^{T}[\Delta m]\{\psi\}_{i}\right)$

Applying Eq. (23) to Eq. (22) we develop an expression that directly relates the physical mode shape changes to the structural changes

$$
\begin{aligned}
\Delta \psi_{k i} & =\sum_{\substack{j=1 \\
j \neq i}}^{n}\left[\frac { \psi _ { k j } } { M _ { j } ( \omega _ { i } ^ { 2 } - \omega _ { j } ^ { 2 } ) } \left(\{\psi\}_{j}^{T}[\Delta k]\{\psi\}_{i}\right.\right. \\
& \left.\left.-\omega_{i}^{2}\{\psi\}_{j}^{T}[\Delta m]\{\psi\}_{i}\right)\right]
\end{aligned}
$$

Using the relationships for the structural changes defined by Eqs. (18) and (19), the first-order perturbation equations for a natural frequency change to the $i$ th mode can be written as

$$
\Delta\left(\omega_{i}^{2}\right)=\frac{1}{M_{i}}\left[\sum_{e=1}^{o}\left(\{\psi\}_{i}^{T}\left[k_{e}\right]\{\psi\}_{i}-\omega_{i}^{2}(\psi\}_{i}^{T}\left[m_{e}\right]\{\psi\}_{i}\right) \alpha_{e}\right]
$$

and for changes to the $k$ th degree of freedom on the $i$ th mode as

$$
\begin{aligned}
& \Delta \psi_{k i}=\sum_{e=1}^{\sigma}\left[\sum _ { \substack { j = 1 \\
j \neq i } } ^ { n } \frac { \psi _ { k j } } { M _ { j } ( \omega _ { i } ^ { 2 } - \omega _ { j } ^ { 2 } ) } \left(\{\psi\}_{j}^{T}\left[k_{e}\right]\{\psi\}_{i}\right.\right. \\
& \left.\left.-\omega_{i}^{2}\{\psi\}_{j}^{T}\left[m_{e}\right](\psi\}_{i}\right)\right] \alpha_{e}
\end{aligned}
$$

Equations (25) and (26) are a set of linear equations with respect to $\alpha_{e}$ that, when solved, will provide a first-order approximation to the structural changes. Should nonlinear expressions be used instead of Eqs. (18) and (19), then Eqs. (25) and (26) would be nonlinear.
The predictor phase problem can be of type 1,2 , or 3 depending on the relation between $\sigma$ and $S$. In any case, the problem solution will yield first-order approximate values to the $\sigma$ element change properties $\alpha_{e}$. The predictor phase algorithm is summarized in Fig. 2.

\section{Corrector Phase}

In the corrector phase, the first-order approximations to the structural changes are used to calculate a first-order approximation to the objective eigenvectors. These approximate objective eigenvectors are used in the general perturbation equations to correct the elemental changes.

The approximate objective eigenvectors are calculated using the linear mapping provided by Eq. (20), where the admixture coefficients $c_{i j}$ are calculated from Eq. (23). With the approximate eigenvectors developed, the general perturbation equations (15), combined with the definitions for structural changes [Eqs. (18) and (19)], are used to develop the following equation:

$$
\begin{aligned}
& \sum_{e=I}^{\sigma}\left(\left\{\psi^{\prime}\right\}_{j}^{T}\left[k_{e}\right]\left\{\psi^{\prime}\right\}_{i}-\omega_{i}{ }^{2}\left\{\psi^{\prime}\right\}_{j}^{T}\left[m_{e}\right]\left\{\psi^{\prime}\right\}_{i}\right) \alpha_{e} \\
& =\left\{\psi^{\prime}\right\}_{j}^{T}[m]\left\{\psi^{\prime}\right\}_{i} \omega_{i}{ }^{2}-\left\{\psi^{\prime}\right\}_{j}^{T}[k]\left\{\psi^{\prime}\right\}_{i}
\end{aligned}
$$

which provides the corrected structural changes. This equation is used for all modes where a frequency constraint is specified. The corrector phase problem can be of type 1,2 , or 3 depending on the relation between $\sigma$ and the number of specified natural frequencies. At the end of the predictor

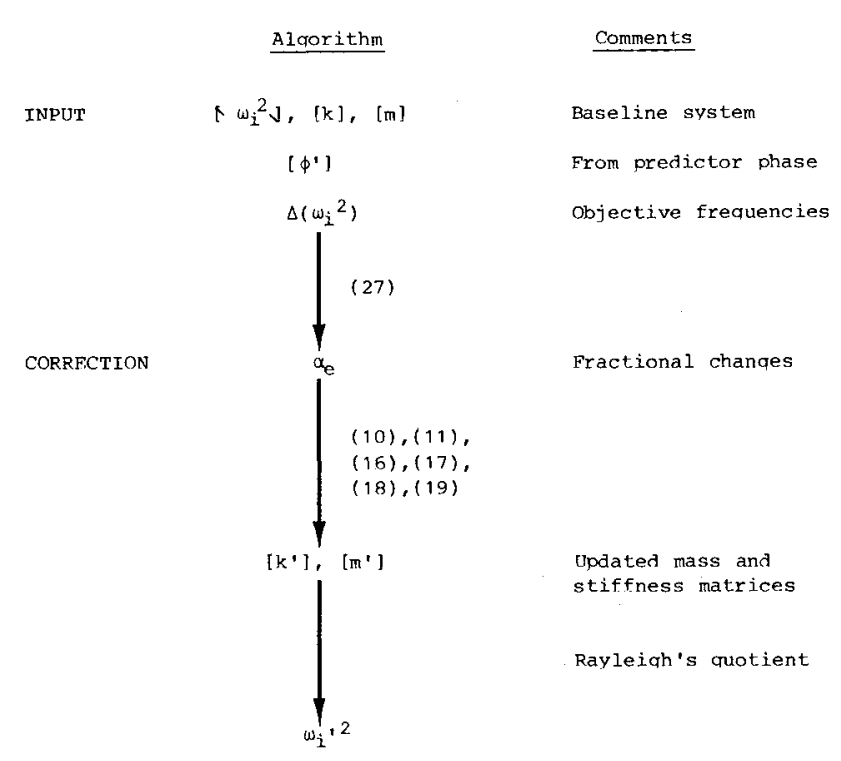

Fig. 3 Corrector phase algorithm.

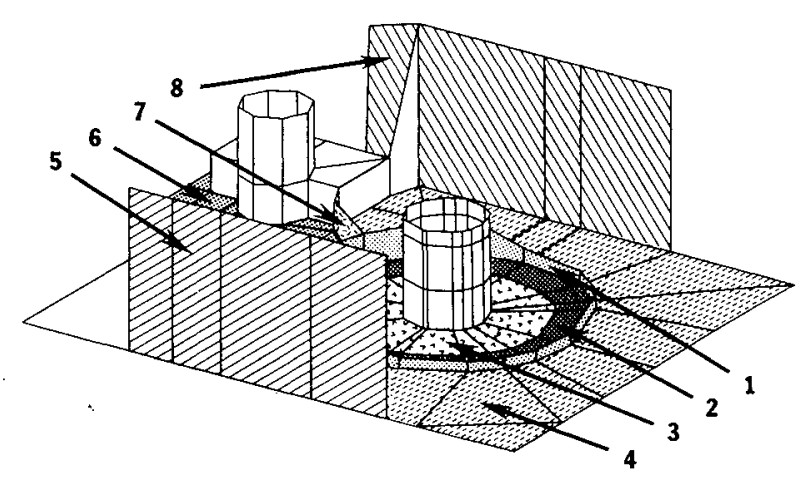

Fig. 4 Disk drive aluminum casting with design parameter sets. 
Table 1 Percentage thickness changes by predictor and corrector phase for each increment

\begin{tabular}{|c|c|c|c|c|c|}
\hline \multirow{2}{*}{$\begin{array}{l}\text { Element } \\
\text { set }\end{array}$} & \multicolumn{4}{|c|}{ Increment No. } & \multirow{2}{*}{$\begin{array}{l}\text { Final thick- } \\
\text { ness design } \\
\text { change } \alpha_{i}\end{array}$} \\
\hline & Predictor & Corrector & Predictor & Corrector & \\
\hline 1 & 7.0 & 6.2 & -0.6 & 12.1 & 19.0 \\
\hline 2 & 6.7 & 16.0 & 2.8 & -17.4 & -4.2 \\
\hline 3 & 14.5 & 39.8 & 2.8 & 13.4 & 58.6 \\
\hline 4 & 10.0 & 10.1 & 5.1 & 15.3 & 26.9 \\
\hline 5 & 8.4 & -4.0 & 3.5 & 13.9 & 9.3 \\
\hline 6 & 7.6 & 58.0 & 4.6 & 20.0 & 89.6 \\
\hline 7 & 2.4 & 0.1 & 2.4 & 7.9 & 8.0 \\
\hline 8 & 6.5 & 9.4 & 1.1 & 6.5 & 16.6 \\
\hline
\end{tabular}

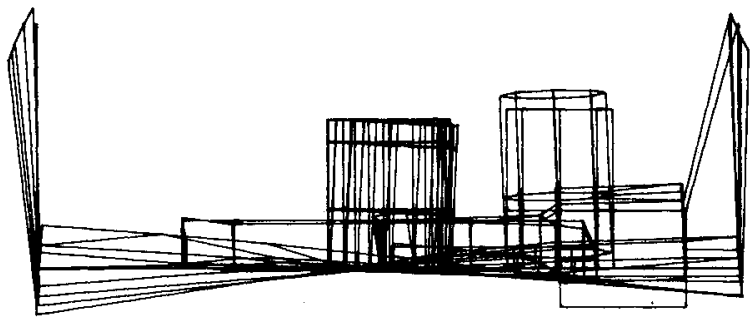

Fig. 5 First mode-floor twist.

phase, however, all the modal degrees of freedom have been computed to first order and are known in the corrector phase problem. Thus, the only unknowns are the $\sigma$ structural changes $\alpha_{e}$ and in practice the corrector phase problem is usually of type 3 . When $i=j$, Eq. (27) enforces the frequency constraint on the $i$ th mode. When $i \neq j$, Eq. (27) is interpreted as enforcing orthogonality between the $i$ th and $j$ th mode shapes.

In the above formulation, only some of the natural frequencies and mode shapes are constrained. If the designer wishes to limit the changes in one of these modes, he should specify upper and lower bounds on their characteristics. The unconstrained modal characteristics, and particularly those of modes close to the constrained modes, are affected in the final design. The corrector phase algorithm is summarized in Fig. 3.

\section{Disk Drive Aluminum Casting Redesign}

The purpose of this example is to redesign the aluminum casting of the Irwin-Olivetti Winchester disk drive to raise the first natural frequency by $30 \%$. Solution of the problem is considered using the design objective of least change from the baseline structure.

\section{Finite Element Model}

It is desired to consider the vibration of the structure as a free body in space. In order to remove the rigid-body motions, the structure is supported by soft springs at the corners.

A total of 312 structural elements were used, involving 209 nodes and 1254 degrees of freedom (Fig. 4). There were 144 beam elements, 8 spring elements, 159 quadrilateral plate elements, and 1 triangular plate element. The quadrilateral and triangular plate elements were used to model the basic casting geometry, while the beam elements were used for the various stiffeners. The spring elements were used to support the structure.

The eigenvalue analysis was performed using the inverse power method option in MSC/NASTRAN. A truncated set of the first eight modes were extracted. The natural modes were normalized by setting the maximum value of each mode to unity. The first natural frequency of the baseline structure occurred at $393.6 \mathrm{~Hz}$. The motion of the structure corresponds to a twisting of the main floor (Fig. 5).

\section{Design Variables}

The design variables for the analysis are the plate thicknesses of the structural elements. Beam and elastic spring elements are held at their baseline values. Variation of only the plate thickness is not considered a significant limitation on the analysis since the plate elements contain over $75 \%$ of the strain energy in the first mode. Design variable linking is employed to link the thickness of several elements to one design parameter. A total of 16 design parameters remain after linking the 160 plate elements. Linking is performed on the basis of similar geometric and structural properties. Manufacturability and perturbation analysis cost considerations are primary reasons for variable linking.

To further reduce the number of significant variables, the strain energy of each one of the 16 parameter sets was calculated. Only sets 1-8 have significant strain energy values for the first mode. Therefore, only these sets are included in the analysis. (See Fig. 4.)

\section{Constraints on Design Variables}

Several constraints are imposed on the design variables to insure a practical design. The thickness of any plate is limited to a minimum of $1.8 \mathrm{~mm}(0.070$ in. $)$ while the maximum thickness is unlimited.

\section{Results}

The analysis was performed using two increments of the predictor-corrector technique. In the first increment a frequency of $470.0 \mathrm{~Hz}$ was achieved, while the second increment achieved the final value of $515.6 \mathrm{~Hz}$. This final value represents a $31 \%$ increase in the first natural frequency or a $3.3 \%$ error in our objective of $511.6 \mathrm{~Hz}$. If the designer wishes to achieve the exact value, another increment could be used. However, this will increase the cost of the analysis.

In the predictor phase the problem was formulated as a type 1 problem with

1) A minimum change objective: $\min \sum_{e}^{o} \alpha_{e}^{2}$.

2) Eight optimization variables $(\sigma=8)$.

3) One equality constraint, that is, Eq. (25) for the first natural frequency.

4) Eight inequality constraints defining the lower bounds for plate thicknesses.

This optimization problem was (a quadratic programming problem) solved using the Nelder and Mead simplex method. ${ }^{15}$

In the corrector phase, the problem was formulated as a type 2 problem with eight unknowns $(\sigma=8)$ and eight equations like Eq. (27) for the first eight modes. The simultaneous solution of the eight equations yielded one solution that did not satisfy the eight inequality constraints defining the lower plate thickness bounds. Consequently, the problem was formulated as a type 3 problem with eight 
unknowns $(\sigma=8)$, eight equations like Eq. (27) for the first eight modes, and eight inequality constraints defining the lower plate thickness bounds. This produced a solution that minimized the error in the equality and inequality constraints.

Results for the predictor and corrector phases of each increment, as well as the total changes, corresponding to each parameter set are shown in Table 1 . In each increment the difference between the predicted changes and the corrected changes is significant. This is interpreted as the adjustment of the predictor phase changes to account for higher-order effects and enforcement of the orthogonality conditions between modes.

\section{Summary}

A nonlinear incremental inverse perturbation method for structural redesign has been developed. The method uses a single finite element analysis of an undamped baseline structural system and can be applied to large or small natural frequency and/or mode shape changes. The redesign problem is solved using an incremental predictor-corrector technique. A 1254-degree-of-freedom aluminum casting has been redesigned for a frequency objective, which was achieved in two steps within $3.3 \%$.

\section{Acknowledgments}

This research has been supported by National Science Foundation Research Grant MEA-8019642 "Inverse Perturbation in Finite Element Dynamic Analysis," and by the Ship Structures Committee. The redesign of the aluminum casting has been funded by the Center for Research in Integrated Manufacturing of The University of Michigan. This support is gratefully acknowledged.

\section{References}

${ }^{1}$ Rayleigh, B., Theory of Sound, Dover Publications, New York, 1945, Vol. 1, pp. 113-118, 214-217.

${ }^{2}$ Turner, M. J., "Design of Minimum Mass Structures with Specified Natural Frequencies," AIAA Journal, Vol. 5, March 1967, pp. 406-412.
${ }^{3}$ Taylor, J. E., "Minimum Mass Bar for Axial Vibration at Specified Natural Frequency," AIAA Journal, Vol. 5, Oct. 1967, pp. 1911-1913.

${ }^{4}$ Sheu, C. Y., "Elastic Minimum-Weight Design for Specified Fundamental Frequency," International Journal of Solids and Structures, Vol. 4, 1968, pp. 953-958.

${ }^{5}$ Pierson, B. L., "A Survey of Optimal Structural Design Under Dynamic Constraints," International Journal of Numerical Methods in Engineering, Vol. 4, No. 4, July-Aug. 1972, pp. 491-499.

${ }^{6}$ Taylor, J. E., "Scaling a Discrete Structural Model to Match Measured Modal Frequencies," AIAA Journal, Vol. 15, Nov. 1977, pp. 1647-1649.

${ }^{7}$ Bellagamba, L. and Yang, T. Y., "Minimum-Mass Truss Structures with Constraints on Fundamental Natural Frequency," AIAA Journal, Vol. 19, Nov. 1981, pp. 1452-1458.

${ }^{8}$ Stetson, K. A., "Perturbation Method of Structural Design Relevant to Holographic Vibration Analysis," AIAA Journal, Vol. 13, April 1975, pp. 457-459.

${ }^{9}$ Stetson, K. A. and Palma, G. E., "Inversion of First-Order Perturbation Theory and Its Application to Structural Design," AIAA Journal, Vol. 14, April 1976, pp. 454-460.

${ }^{10}$ Stetson, K. A., Harrison, I. R., and Palma, G. E., "Redesigning Structural Vibration Modes by Inverse Perturbation Subject to Minimal Change Theory," Computer Methods in Applied Mechanics and Engineering, Vol. 16, 1978, pp. 151-175.

${ }^{11}$ Stetson, K. A. and Harrison, I. R., "Redesign of Structural Vibration Modes by Finite-Element Inverse Perturbation,"' Journal of Engineering for Power, Vol. 103, April 1981, pp. 319-325.

${ }^{12}$ Sandström, R. E., "Inverse Perturbation Methods for Vibration Analysis," Proceedings, NATO Advanced Study Institute on Optimization of Distributed Parameter Structural Systems, University of Iowa, Iowa City, May 20-June 4, 1980, pp. 1539-1552.

${ }^{13}$ Sandström, R. E. and Anderson, W. J., "Modal Perturbation Methods for Marine Structures," Transactions of the Society of Naval Architects and Marine Engineers, Vol. 90, 1982, pp. 41-54.

${ }^{14}$ Kim, K. O., Anderson, W. J., and Sandström, R. E., "Nonlinear Inverse Peturbation Method in Dynamic Analysis," AIAA Journal, Vol. 21, Sept. 1983, pp. 1310-1316.

${ }^{15}$ Parsons, M. G., "Optimization Methods for use in ComputerAided Ship Design," Paper presented at First Ship Technology and Research Symposium, Washington, D.C., Aug. 26-29, 1975. 